\title{
Cartan's concept of duality for second-order ordinary differential equations
}

\author{
M. Crampin ${ }^{\mathrm{a}, *}$, D.J. Saunders ${ }^{\mathrm{b}}$ \\ ${ }^{a}$ Department of Mathematical Physics and Astronomy, Ghent University, Krijgslaan 281, \\ B-9000 Gent, Belgium \\ b Department of Applied Mathematics, The Open University, Walton Hall, \\ Milton Keynes MK7 6AA, UK
}

Received 28 April 2004; received in revised form 13 September 2004; accepted 24 September 2004 Available online 11 November 2004

\begin{abstract}
We discuss the theory of the second-order ordinary differential equation initiated by Cartan, concentrating especially on Cartan's notion of duality between such equations, and its consequences. (C) 2004 Elsevier B.V. All rights reserved.
\end{abstract}

PACS: 02.40

MSC: $34 \mathrm{~A} 26$

1JGP SC: Geometry theory of differential equations

Keywords: Cartan projective connection; Second-order ordinary differential equation; Duality; CR structure

\section{Introduction}

There has been a flurry of interest recently among some relativists in Cartan's theory of the second-order ordinary differential equation, to be found in his paper of 1924 [3]

\footnotetext{
* Corresponding author. Present address: 65 Mount Pleasant, Aspley Guise, Beds MK17 8JX, UK.

E-mail address: crampin@btinternet.com (M. Crampin).
} 
on projective connections. The origin of this interest can be traced to a paper of Newman and co-workers [7]; in the introduction to this paper the authors describe how they discovered, to their surprise, that work of Cartan on the invariants of differential equations was relevant to their programme of reformulating general relativity in terms of null surfaces. The relevant study is in fact concerned with third-order ordinary differential equations, and was carried out by Chern using Cartan's methods; moreover, the relevance is to the conformal geometry of three-dimensional rather than four-dimensional Lorentzian manifolds. Nevertheless Newman and co-workers found this discovery to be fruitful, and were able to generalize the approach of Cartan and Chern so as to apply it to the full four-dimensional theory.

There is a general philosophy at work here, that it is possible to construct certain kinds of geometric structures on the spaces of solutions of suitable types of differential equations. Thus in the case of the third-order ordinary differential equation one is able to create from the equation a conformal class of Lorentzian metrics on the solution space, provided that a certain function associated with the equation vanishes; this function is a relative invariant of the equation under contact transformations, and is known as the Wünschmann invariant after its discoverer.

Constructions of this kind are both useful and intriguing, and it is natural therefore that they should be studied as objects of interest in their own right. The simplest case is that of the second-order ordinary differential equation. Two papers on this topic have appeared recently. The first [8] deals directly with the kind of question just described, namely the construction of a geometric structure on the solution space of a second-order ordinary differential equation and the identification of a function of Wünschmann type, here a relative invariant of the equation under point transformations. The second [9] examines the relation between the geometry of a second-order ordinary differential equation and that of a Cauchy-Riemann structure from a somewhat similar point of view, and describes the construction of a conformal class of split-signature four-dimensional metrics associated with each second-order ordinary differential equation, analogous to the Fefferman metrics associated with Cauchy-Riemann structures.

Cartan's seminal influence on these studies is universally acknowledged; but it seems fair to say that that influence is not always completely understood. His paper on projective connections is a case in point. The paper falls into two parts, the first and larger of which deals in effect with the projective differential geometry of affine connections, or equivalently with systems of second-order ordinary differential equations of geodesic type, and the second with the geometry of a single second-order ordinary differential equation $\mathrm{d}^{2} y / \mathrm{d} x^{2}=$ $f(x, y, \mathrm{~d} y / \mathrm{d} x)$ with no such restriction as to its type; we should make it clear that we shall be concerned here almost entirely with the second part of the paper. In it Cartan shows how to construct what he calls a normal projective connection associated with the equation, whose curvature has in effect two components, which he calls $a$ and $b$; they are relative invariants of the equation under point transformations (coordinate transformations of the form $\hat{x}=\phi(x, y), \hat{y}=\psi(x, y))$. The vanishing of $a$ is the necessary and sufficient condition for the equation to be equivalent to a system of affine geodesic type on the two-dimensional manifold whose coordinates are $x$ and $y$; the normal projective connection associated with the differential equation then reduces to that of the affine type associated with the geodesic system as described in the first part of the paper. Given that $a=0$, the vanishing of $b$ is 
the necessary and sufficient condition for the equation to be rectifiable, that is, reducible to the form $\mathrm{d}^{2} \hat{y} / \mathrm{d} \hat{x}^{2}=0$ by a point transformation. So much is to be found in Cartan's paper. There remains unanswered an obvious question, namely what is the significance of $b$ independent of the vanishing of $a$ ?

This question can be answered in a couple of different ways. One answer is to identify $b$ in terms of the projectively invariant tensors associated with the projective equivalence class of sprays associated with the equation; this will be explained briefly below, and is dealt with at greater length in [4]. The other, which is the one of interest here, is in effect provided in [8]: the geometric structure on the solution space of the equation sought therein is a projective connection of affine type, and $b$ turns out to be the corresponding Wünschmann-like invariant, whose vanishing is the condition for such a structure to exist. We can express this result as follows (similar accounts have been given in [2,9]). The second-order differential equation should be considered as a line-element field (a vector field determined up to a scalar factor) on PTM, the projective tangent bundle of the two-dimensional manifold $M$ of coordinates $x$ and $y$. The solution space is the base space of the fibration of PTM it defines. Thus PTM has a double fibration with one-dimensional fibres. Either can be thought of as defining a differential equation; we therefore have two equations, which we describe as dual to each other. Interchanging the roles of the fibrations has the effect of interchanging the roles of $a$ and $b$; thus $b=0$ is the condition for the dual equation to be of affine geodesic type.

This notion of duality is discussed by Cartan himself in a single rather brief and not very transparent section of [3]; the significance of the vanishing of $b$ just described is not made explicit there, though it is a simple consequence of what is said. It is derived in Appendix A to [7], in an argument due to Tod which owes nothing to Cartan's approach. It is also derived in [8]; here the methods used are closer to Cartan's in general, but still they differ significantly from those of the relevant section of [3].

Given the current interest in Cartan's results, it seems timely to explain them in his terms as an alternative to reconstructing them by other means, and this we shall do here for duality of second-order ordinary differential equations. One unexpected result of our analysis is that Cartan's account appears to be wrong in some details, if it is not lèse-majesté to say so. As well as clarifying such points, we are able to throw a somewhat different light on the relation between the geometry of a differential equation and CR geometry from that described in [9] (though we should make it clear that we do not discuss here the Fefferman metrics).

One difficulty the modern reader faces with Cartan's writings on connections is that his idea of a connection is mathematically subtly different, and conceptually very different, from the much more familiar one of Ehresmann. Fortunately there is now a good modern account of Cartan's theory of connections due to Sharpe [10]; we briefly describe the relevant parts of the theory from Sharpe's point of view in Section 2. In Section 3 we explain Cartan's construction of the normal projective connection associated with a differential equation. The theory of duality is discussed in a manner close to Cartan's in Section 4. In Section 5 we reconsider the matter, treating the two fibrations on a more equal footing than before. The results we obtain in this way are immediately transferable to the study of CR structures, as we describe in Section 6. We have reprinted the section of Cartan's paper which deals with duality as Appendix A, for ease of reference. 


\section{Cartan projective connections}

As we noted above, Cartan's view of a connection is rather different from that formalized later by Ehresmann. In the case of a projective connection it involves a manifold with a projective space attached to each point, and the different projective spaces are 'connected' to one another by an infinitesimal relationship. The modern view of this concept described by Sharpe [10] relates it to Klein's notion of geometry, which in effect proposes that a geometry should be considered as a homogeneous space of a Lie group.

Let $\mathfrak{G}$ be a Lie group with Lie algebra $\mathfrak{g}$. The infinitesimal properties of the multiplication on $\mathfrak{G}$ are encoded in its Maurer-Cartan form, a $\mathfrak{g}$-valued left-invariant 1-form $\tilde{\omega}$ defined by setting $\langle\tilde{X}, \tilde{\omega}\rangle=X$ for any $X \in \mathfrak{g}$, where $\tilde{X}$ is the left-invariant vector field on $\mathfrak{G}$ corresponding to $X$. Then for each $g \in \mathfrak{G}, \tilde{\omega}_{g}: T_{g} \mathfrak{G} \rightarrow \mathfrak{g}$ is an isomorphism, and $\tilde{\omega}$ also satisfies the important properties that $R_{g}^{*} \tilde{\omega}=\operatorname{ad}\left(g^{-1}\right) \tilde{\omega}$ and that $\mathrm{d} \tilde{\omega}+(1 / 2)[\tilde{\omega} \wedge \tilde{\omega}]=0$. If $\mathfrak{G}$ is a matrix group then we may write $\tilde{\omega}$ as $g^{-1} \mathrm{~d} g$, and this often simplifies the notation; we shall henceforth assume that $\mathfrak{G}$ is a matrix group.

A Klein geometry is then a homogeneous space of $\mathfrak{G}$, so that it is a manifold $M$ with a transitive left action of $\mathfrak{G}$; we shall also suppose that the action is effective. Take a point $\xi_{0} \in M$, and let $\mathfrak{H}$ be the stabilizer of $\xi_{0}$; then $M$ can be identified with the coset space $\mathfrak{G} / \mathfrak{H}$. In this way, $\mathfrak{G}$ becomes a right principal $\mathfrak{H}$-bundle over $M$ with projection $g \mapsto g \xi_{0}$; we may alternatively refer to the pair $(\mathfrak{G}, \mathfrak{H})$ as the Klein geometry.

In the description given by Sharpe, a Cartan geometry on a manifold $M$ is modelled on a Klein geometry $(\mathfrak{G}, \mathfrak{H})$. It is a right principal $\mathfrak{H}$-bundle $\pi: P \rightarrow M$ of the correct dimension (that is, $\operatorname{dim} P=\operatorname{dim} \mathfrak{G}=\operatorname{dim} \mathfrak{H}+\operatorname{dim} M$ ) together with a $\mathfrak{g}$-valued 1 -form $\omega$ on $P$, the Cartan connection form, sharing as many of the properties of the Maurer-Cartan form $\tilde{\omega}$ as possible:

- if $\mathfrak{h}$ is the Lie algebra of $\mathfrak{H}$ and $X \in \mathfrak{h}$ then $\langle\tilde{X}, \omega\rangle=X$, where $\tilde{X}$ is the vertical vector field on $P$ generated by $X$ through the action of $\mathfrak{H}$;

- for each $p \in P, \omega_{p}: T_{p} P \rightarrow \mathfrak{g}$ is an isomorphism;

- for each $h \in \mathfrak{H}, R_{h}^{*} \omega=\operatorname{ad}\left(h^{-1}\right) \omega$.

One property of the Maurer-Cartan form which is not required to hold in this more general context is the vanishing of $\mathrm{d} \omega+(1 / 2)[\omega \wedge \omega]$, and indeed the curvature $\Omega$ of a Cartan connection is defined to be the $\mathfrak{g}$-valued 2 -form

$$
\Omega=\mathrm{d} \omega+\frac{1}{2}[\omega \wedge \omega]
$$

for a matrix Lie algebra the components of the curvature matrix may be written as $\Omega_{j}^{i}=$ $\mathrm{d} \omega_{j}^{i}+\omega_{k}^{i} \wedge \omega_{j}^{k}$. The vanishing of the curvature is then a necessary and sufficient condition for the Cartan geometry to be locally isomorphic to the Klein geometry on which it is modelled. In addition, the curvature satisfies the Bianchi identity $\mathrm{d} \Omega=[\Omega \wedge \omega]$, and the torsion of the connection is defined to be the $\mathfrak{g} / \mathfrak{h}$-valued 2-form $\rho(\Omega)$, where $\rho: \mathfrak{g} \rightarrow \mathfrak{g} / \mathfrak{h}$ is the projection. 
An important concept in a Klein geometry is that of the development of a curve. A curve in the Lie algebra $\mathfrak{g}$ can be 'developed' to a curve in the homogeneous space $\mathfrak{G} / \mathfrak{H}$ through any given point, in the following way. If $t \mapsto X(t)$ is the curve in $\mathfrak{g}$, and $g_{0} \in \mathfrak{G}$, there is a unique curve $t \mapsto g(t)$ in $\mathfrak{G}$ such that $\langle\dot{g}(t), \tilde{\omega}\rangle=X(t)$ and $g(0)=g_{0} ; g(t)$ is a solution of the differential equation $\dot{g}=g X$. The development of $X(t)$ into $\mathfrak{G} / \mathfrak{H}$ through $\xi=g_{0} \mathfrak{H}$ is then defined to be the curve $\xi(t)=\mathfrak{g}(t) \mathfrak{H}$ in $\mathfrak{G} / \mathfrak{H}$. If $\gamma$ is a local section of $\mathfrak{G}$ over some neighbourhood of $\xi$ in $\mathfrak{G} / \mathfrak{H}$, then the curves $g(t)$ (in $\mathfrak{G}$ ) and $\xi(t)$ (in $\mathfrak{G} / \mathfrak{H}$ ) are related by $g(t)=\gamma(\xi(t)) h(t)$ for some curve $t \mapsto h(t)$ in $\mathfrak{H}$; the curves $\xi(t)$ and $h(t)$ satisfy the differential equation

$$
h^{-1} \dot{h}+h^{-1}\left\langle\dot{\xi}, \gamma^{*} \tilde{\omega}\right\rangle h=g^{-1} \dot{g}=X
$$

The notion of development is also appropriate for a Cartan geometry. If $t \mapsto p(t)$ is a curve in $P$ then $t \mapsto\left\langle\dot{p}(t), \omega_{p(t)}\right\rangle$ is a curve in $\mathfrak{g}$ which can be developed into $\mathfrak{G} / \mathfrak{H}$ as before; this development is $\xi(t)=g(t) \mathfrak{H}$ where $\langle\dot{g}, \tilde{\omega}\rangle=\langle\dot{p}, \omega\rangle$. But $\tilde{\omega}$ and $\omega$ transform identically under the action of $\mathfrak{H}$, which means that $\xi(t)$ depends only on $\pi(p(t))$; thus a curve in $M$ and a point in $\mathfrak{G} / \mathfrak{H}$ determine a curve in $\mathfrak{G} / \mathfrak{H}$ starting at the given point, called a development of the curve in $M$ into $\mathfrak{G} / \mathfrak{H}$. If the Klein geometry contains straight lines, a curve in $M$ is called a geodesic of the Cartan geometry if its development through any point is a straight line.

For the purposes of calculation it is convenient to choose a gauge, in other words a local section $\sigma$ of $P$, as we can then study a connection using forms on $M$; if these transform correctly under change of gauge then we can always recover the connection form on the larger manifold $P$ if required. The gauged Cartan connection form is $\sigma^{*} \omega$, so that this is a $\mathfrak{g}$-valued local 1-form on $M$ with the properties that $\left.\sigma^{*} \omega\right|_{x}$ is an injective map $T_{x} M \rightarrow \mathfrak{g}$, and that $\left.\rho \circ \sigma^{*} \omega\right|_{x}: T_{x} M \rightarrow \mathfrak{g} / \mathfrak{h}$ is an isomorphism. If $\hat{\sigma}$ is another gauge then on the intersection of their domains $\hat{\sigma}(x)=\sigma(x) h(x)$ for some $\mathfrak{H}$-valued function $h$; the transformation rules are then

$$
\hat{\sigma}^{*} \omega=h^{-1}\left(\sigma^{*} \omega\right) h+h^{-1} \mathrm{~d} h, \quad \hat{\sigma}^{*} \Omega=h^{-1}\left(\sigma^{*} \Omega\right) h .
$$

The differential equation for a development, when expressed in the gauge $\sigma$, becomes

$$
h^{-1} \dot{h}+h^{-1}\left\langle\dot{\xi}, \gamma^{*} \tilde{\omega}\right\rangle h=\left\langle\dot{x}, \sigma^{*} \omega\right\rangle
$$

this comprises $\operatorname{dim} \mathfrak{g}$ equations for $\operatorname{dim}(\mathfrak{g} / \mathfrak{h})$ unknowns $\xi$ and $\operatorname{dim} \mathfrak{h}$ unknowns $h$.

We shall (as did Cartan) use a gauge in our calculations below, and omit the $\sigma^{*}$; a significant part of the procedure is to make successive changes of gauge in order to simplify the gauged connection and curvature forms.

\section{The manifold of elements}

Our particular concern in this paper is with what Cartan calls a 'manifold of elements with projective connection' in the two-dimensional case. An element is a pair consisting of 
a point of a differentiable manifold $M$ and a one-dimensional subspace of the tangent space to $M$ at that point. Thus a manifold of elements is the projective tangent bundle PTM of a two-dimensional manifold $M$. We denote by $\mathrm{P}^{2}$ real projective space of two dimensions; its projective tangent bundle $\mathrm{P}^{2} \mathrm{P}^{2}$ can be expressed as the homogeneous space $\mathfrak{G} / \mathfrak{H}$ where $\mathfrak{G}=\operatorname{SL}(3, \mathbf{R})$ and $\mathfrak{H}$ is the subgroup of $\mathfrak{G}$ consisting of its upper triangular elements. A manifold of elements with projective connection is a Cartan geometry on PTM modelled on $\mathrm{PTP}^{2}$, in which certain conditions regarding the development of curves, arising out of the projective tangent structures of the underlying manifold and the model geometry, are satisfied; we shall describe these conditions shortly.

Before doing so, however, we must deal with various matters arising from the basic definition. First, we point out that one can introduce local coordinates on PTM by taking local coordinates $(x, y)$ on $M$, and by noting that every equivalence class of tangent vectors

$$
u \frac{\partial}{\partial x}+v \frac{\partial}{\partial y}
$$

for which $u \neq 0$ has a unique representative of the form

$$
\frac{\partial}{\partial x}+y^{\prime} \frac{\partial}{\partial y}
$$

then $\left(x, y, y^{\prime}\right)$ are local coordinates on PTM, and we shall always work with such coordinates, while recognising that they do not cover those equivalence classes of tangent vectors for which $u=0$.

Next, we make some remarks about changes of gauge for a projective connection on such a manifold. If $\omega$ is a connection form - a trace-free $3 \times 3$ matrix of local 1-forms on PTM - and $H$ an $\mathfrak{H}$-valued function, then the connection form regauged by $H$ is

$$
H^{-1} \omega H+H^{-1} \mathrm{~d} H
$$

and if $\Omega$ is the curvature 2-form corresponding to $\omega$ then the regauged curvature is $H^{-1} \Omega H$. If

$$
H=\left[\begin{array}{lll}
A & D & F \\
0 & B & E \\
0 & 0 & C
\end{array}\right]
$$

with $A B C=1$, then

$$
H^{-1}=\left[\begin{array}{ccc}
A^{-1} & -C D & D E-B F \\
0 & B^{-1} & -A E \\
0 & 0 & C^{-1}
\end{array}\right]
$$


It is the effect of a change of gauge on the strictly lower triangular terms in $\omega$ that is of immediate interest. Since $H^{-1} \mathrm{~d} H$ is upper triangular it has no effect on these terms. Suppose that

$$
\omega=\left[\begin{array}{lll}
* & * & * \\
u & * & * \\
w & v & *
\end{array}\right],
$$

then

$$
H^{-1} \omega H=\left[\begin{array}{ccc}
* & * & * \\
u^{\prime} & * & * \\
w^{\prime} & v^{\prime} & *
\end{array}\right],
$$

where

$$
u^{\prime}=A B^{-1} u-A^{2} E w, \quad v^{\prime}=B C^{-1} v+C^{-1} D w, \quad w^{\prime}=A C^{-1} w .
$$

The effect of a change of gauge on a curvature form which is strictly upper triangular will be of interest later. If

$$
\Omega=\left[\begin{array}{lll}
0 & U & * \\
0 & 0 & V \\
0 & 0 & 0
\end{array}\right],
$$

then

$$
H^{-1} \Omega H=\left[\begin{array}{ccc}
0 & U^{\prime} & * \\
0 & 0 & V^{\prime} \\
0 & 0 & 0
\end{array}\right],
$$

where

$$
U^{\prime}=A^{-1} B U, \quad V^{\prime}=B^{-1} C V .
$$

We shall also need the equations for the development of a curve. It is easy to see that

$$
\left(\xi, \eta, \eta^{\prime}\right) \mapsto\left[\begin{array}{ccc}
1 & 0 & 0 \\
\xi & 1 & 0 \\
\eta & \eta^{\prime} & 1
\end{array}\right]
$$


is a local section of $\mathrm{SL}(3, \mathbf{R}) \rightarrow \mathrm{PTP}^{2}$, and that the corresponding gauged Maurer-Cartan form is

$$
\left[\begin{array}{ccc}
0 & 0 & 0 \\
\mathrm{~d} \xi & 0 & 0 \\
\mathrm{~d} \eta-\eta^{\prime} \mathrm{d} \xi & \mathrm{d} \eta^{\prime} & 0
\end{array}\right] .
$$

Following Cartan, we shall write the connection form $\omega$ explicitly as

$$
\omega=\left[\begin{array}{lll}
\omega_{0}^{0} & \omega_{1}^{0} & \omega_{2}^{0} \\
\omega^{1} & \omega_{1}^{1} & \omega_{2}^{1} \\
\omega^{2} & \omega_{1}^{2} & \omega_{2}^{2}
\end{array}\right] .
$$

The development equations for a curve $\gamma$ in PTM give

$$
a \dot{\xi}-b\left(\dot{\eta}-\eta^{\prime} \dot{\xi}\right)=\left\langle\dot{\gamma}, \omega^{1}\right\rangle, \quad c\left(\dot{\eta}-\eta^{\prime} \dot{\xi}\right)=\left\langle\dot{\gamma}, \omega^{2}\right\rangle
$$

for some functions $a(t), b(t), c(t)$.

Having established these formulae, we return to the conditions we shall impose on the development of a curve. They concern two particular kinds of curve on PTM: vertical curves and natural lifts. With respect to the coordinates $\left(x, y, y^{\prime}\right)$ described above, a curve in PTM is vertical if its tangent vector is annihilated by $\mathrm{d} x$ and $\mathrm{d} y$, and a curve in PTM is a natural lift if its tangent vector is annihilated by the contact form $\mathrm{d} y-y^{\prime} \mathrm{d} x$. (Cartan calls a tangent vector to a natural lift a 'multiplicity'.) Our conditions are that

- the development into $\mathrm{PTP}^{2}$ of a vertical curve in $\mathrm{P} T M$ is vertical;

- the development into $\mathrm{PTP}^{2}$ of a natural lift in $\mathrm{P} T M$ is a natural lift.

These conditions therefore require that if $\gamma$ is vertical then $\left\langle\dot{\gamma}, \omega^{1}\right\rangle=\left\langle\dot{\gamma}, \omega^{2}\right\rangle=0$, while if $\gamma$ is a natural lift then $\left\langle\dot{\gamma}, \omega^{2}\right\rangle=0$. It follows that

$$
\omega^{1}=\lambda \mathrm{d} x+\mu \mathrm{d} y, \quad \omega^{2}=v\left(\mathrm{~d} y-y^{\prime} \mathrm{d} x\right)
$$

for some functions $\lambda, \mu$ and $v$ on PTM.

We can now simplify the connection matrix $\omega$ using a change of gauge. By setting

$$
A=\left(\left(\lambda+y^{\prime} \mu\right) \nu\right)^{-1 / 3}, \quad B=\left(\lambda+y^{\prime} \mu\right) A, \quad C=\nu A, \quad E=\mu A,
$$

we can make $\omega^{1}=\mathrm{d} x$ and $\omega^{2}=\mathrm{d} y-y^{\prime} \mathrm{d} x=\theta$. We can also write $\omega_{1}^{2}$ as $\omega_{1}^{2}=k\left(\mathrm{~d} y^{\prime}-\right.$ $f \mathrm{~d} x)+l \theta$ for some functions $f, k$ and $l$ on PTM, and the coefficient $k$ must be non-zero since the forms $\omega^{1}, \omega^{2}$ and $\omega_{1}^{2}$ must be linearly independent. Set $\mathrm{d} y^{\prime}-f \mathrm{~d} x=\phi$; the forms $\mathrm{d} x, \theta$ and $\phi$ constitute a local basis of 1 -forms, and we shall generally carry out calculations in this basis, in this section and the next. 
So far, therefore, we have chosen a gauge such that

$$
\omega=\left[\begin{array}{ccc}
\omega_{0}^{0} & * & * \\
\mathrm{~d} x & * & * \\
\theta & k \phi+l \theta & *
\end{array}\right]
$$

The remaining gauge freedom involves the functions $D$ and $F$, and so with a further gauge change using

$$
H=\left[\begin{array}{lll}
1 & D & F \\
0 & 1 & 0 \\
0 & 0 & 1
\end{array}\right]
$$

we obtain

$$
H^{-1} \omega H+H^{-1} \mathrm{~d} H=\left[\begin{array}{ccc}
\omega_{0}^{0}-D \mathrm{~d} x-F \theta & * & * \\
\mathrm{~d} x & * & * \\
\theta & k \phi+l^{\prime} \theta & *
\end{array}\right]
$$

We may therefore choose the gauge for any projective connection on a manifold of elements so that

$$
\omega^{1}=\mathrm{d} x, \quad \omega^{2}=\theta, \quad \omega_{0}^{0}=\kappa \phi
$$

for some function $\kappa$. Let us call this the standard gauge for the projective connection. It differs from Cartan's choice of standard gauge in the way we have chosen to use the remaining gauge freedom after fixing $\omega^{1}$ and $\omega^{2}$; our choice generalizes more readily to higher dimensional cases, as we shall show elsewhere [5].

A geodesic of this projective connection is a curve whose development satisfies $\dot{\eta}-\eta^{\prime} \dot{\xi}=$ 0 and $\dot{\eta}^{\prime}=0$; that is, a geodesic is a curve whose tangents are annihilated by both $\theta$ and $\phi$, and is therefore a solution of the second-order differential equation

$$
\frac{\mathrm{d}^{2} y}{\mathrm{~d} x^{2}}=f\left(x, y, \frac{\mathrm{d} y}{\mathrm{~d} x}\right) .
$$

Thus geodesics are the base integral curves of the vector field

$$
\Gamma=\frac{\partial}{\partial x}+y^{\prime} \frac{\partial}{\partial y}+f \frac{\partial}{\partial y^{\prime}}
$$


on PTM, the 'second-order differential equation field' corresponding to the projective connection. Note that $\Gamma$ is determined by the conditions

$$
\langle\Gamma, \mathrm{d} x\rangle=1, \quad\langle\Gamma, \theta\rangle=\langle\Gamma, \phi\rangle=0 .
$$

Under a change of coordinates on the base manifold $M$, with the induced change on PTM, $\Gamma$ will acquire an overall factor (depending on the coordinate transformation functions) - so we are really working, not with a vector field $\Gamma$, but with a line-element field $\langle\Gamma\rangle$ say.

Having fixed the gauge, the next step is to impose gauge-invariant conditions on the curvature in order to single out a particular connection from the class of connections being considered. Cartan shows in effect that there is a unique choice of the remaining connection forms so that the curvature $\Omega$ is strictly upper triangular with $\Omega_{1}^{0}$ a multiple of $\mathrm{d} x \wedge \theta$. (In fact Cartan does not go quite as far as this: his connection is determined only up to the addition of a (1-form) multiple of the identity matrix, and his curvature is upper triangular with equal diagonal elements. However, the ambiguity in $\omega$ can be eliminated by insisting that it takes its values in $\mathfrak{s l}(3, \mathbf{R})$, that is, that it be trace-free; the curvature will then be trace-free also. Cartan in effect works with the projective group considered as $\operatorname{GL}(3, \mathbf{R})$ modulo multiples of the identity.) The unique connection obtained in this way is called by Cartan the normal projective connection on the manifold of elements, associated with the second-order differential equation.

A calculation equivalent to Cartan's, but differing from his in that it is carried out in terms of our gauge rather than his, leads to the following result. Given a second-order differential equation, represented by a line element field $\langle\Gamma\rangle$, among the projective connections with the base integral paths of $\langle\Gamma\rangle$ as geodesics there is a unique one whose curvature form $\Omega$ is strictly upper triangular with $\Omega_{1}^{0}$ semi-basic, that is, a multiple of $\mathrm{d} x \wedge \mathrm{d} y=\mathrm{d} x \wedge \theta$. In our standard gauge the $\mathfrak{s l}(3, \mathbf{R})$-valued connection form $\omega$ of this connection is given explicitly in terms of the basis of 1 -forms $\{\mathrm{d} x, \theta, \phi\}$ and the vector field $\Gamma$ by

$$
\omega=\left[\begin{array}{ccc}
0 & \xi \mathrm{d} x+\rho \theta & \rho \mathrm{d} x+\rho_{y^{\prime}} \theta \\
\mathrm{d} x & \frac{1}{3} f_{y^{\prime}} \mathrm{d} x+\frac{1}{6} f_{y^{\prime} y^{\prime}} \theta & \frac{1}{6} f_{y^{\prime} y^{\prime}} \mathrm{d} x+\frac{1}{6} f_{y^{\prime} y^{\prime} y^{\prime}} \theta \\
\theta & \phi-\frac{1}{3} f_{y^{\prime}} \theta & -\frac{1}{3} f_{y^{\prime}} \mathrm{d} x-\frac{1}{6} f_{y^{\prime} y^{\prime}} \theta
\end{array}\right]
$$

where

$$
\xi=f_{y}+\frac{2}{9} f_{y^{\prime}}^{2}-\frac{1}{3} \Gamma\left(f_{y^{\prime}}\right), \quad \rho=\frac{1}{3} f_{y y^{\prime}}+\frac{1}{18} f_{y^{\prime}} f_{y^{\prime} y^{\prime}}-\frac{1}{6} \Gamma\left(f_{y^{\prime} y^{\prime}}\right)
$$

and the subscripts denote partial derivatives; the other coefficient, $\rho_{y^{\prime}}$, can be expressed as

$$
\rho_{y^{\prime}}=\frac{1}{6} f_{y y^{\prime} y^{\prime}}+\frac{1}{18} f_{y^{\prime} y^{\prime}}^{2}-\frac{1}{9} f_{y^{\prime}} f_{y^{\prime} y^{\prime} y^{\prime}}-\frac{1}{6} \Gamma\left(f_{y^{\prime} y^{\prime} y^{\prime}}\right)
$$

These somewhat formidable looking expressions can be written in relatively simple form in terms of the fundamental invariants (as Douglas [6] calls them) of the projective equivalence class of sprays associated with $\Gamma$, as we shall show elsewhere [5]. 
A further calculation reveals the important fact that $\Omega_{2}^{1}$ is a scalar multiple of $\theta \wedge \phi$ alone: in fact

$$
\Omega_{2}^{1}=-\frac{1}{6} f_{y^{\prime} y^{\prime} y^{\prime} y^{\prime}} \theta \wedge \phi
$$

Thus

$$
\Omega=\left[\begin{array}{ccc}
0 & b \mathrm{~d} x \wedge \theta & * \\
0 & 0 & a \theta \wedge \phi \\
0 & 0 & 0
\end{array}\right],
$$

where $a=-(1 / 6) f_{y^{\prime} y^{\prime} y^{\prime} y^{\prime}}$. Cartan says that it is pointless to calculate $b$, and since it is given by

$$
\begin{aligned}
b= & \frac{1}{6} f^{2} f_{y^{\prime} y^{\prime} y^{\prime} y^{\prime}}+\frac{1}{3} f f_{x y^{\prime} y^{\prime} y^{\prime}}+\frac{1}{3} y^{\prime} f f_{y y^{\prime} y^{\prime} y^{\prime}}+\frac{1}{6} f_{x x y^{\prime} y^{\prime}}+\frac{1}{3} y^{\prime} f_{x y y^{\prime} y^{\prime}} \\
& +\frac{1}{6} y^{\prime 2} f_{y y y^{\prime} y^{\prime}}+\frac{1}{6}\left(f_{x}+y^{\prime} f_{y}\right) f_{y^{\prime} y^{\prime} y^{\prime}}-\frac{1}{6} f_{y^{\prime}} f_{x y^{\prime} y^{\prime}}-\frac{1}{6}\left(3 f+y^{\prime} f_{y^{\prime}}\right) f_{y y^{\prime} y^{\prime}} \\
& -\frac{2}{3} f_{x y y^{\prime}}-\frac{2}{3} y^{\prime} f_{y y y^{\prime}}-\frac{1}{2} f_{y} f_{y^{\prime} y^{\prime}}+\frac{2}{3} f_{y^{\prime}} f_{y y^{\prime}}+f_{y y},
\end{aligned}
$$

one sees his point. However, direct comparison of this expression with one given by Shen in [11] shows that it is essentially one of the two basic projectively invariant tensors associated with $\Gamma$, the one first defined by Berwald [1]. The coefficient $a$, on the other hand, determines the Douglas tensor, the other projectively invariant tensor [6]. The vanishing of the Douglas tensor (and equivalently the vanishing of $a$ ) is the necessary and sufficient condition for $\Gamma$ to be projectively equivalent to an affine spray; when this holds (in two dimensions), the vanishing of the Berwald tensor (and equivalently the vanishing of $b$ ) is the necessary and sufficient condition for $\Gamma$ to be projectively flat, or rectifiable.

The remaining entry in the curvature form, $\Omega_{2}^{0}$, is not of much concern because it is completely determined by $a$ and $b$ by means of the Bianchi identity.

Results essentially equivalent to those of Cartan described above are obtained in [9], but by the use of Cartan's method of equivalence rather than by direct consideration of the connection as in [3].

\section{Duality}

Cartan states that 'the idea of element in projective geometry is self-dual, as is the idea of multiplicity' (see Appendix A). We next explain these remarks.

Two-dimensional real projective space $\mathrm{P}^{2}$ is the space of rays in $\mathbf{R}^{3}$. For $[X] \in \mathrm{P}^{2}$, where $[X]$ is the ray through the point $X \in \mathbf{R}^{3}-\{0\}$, the projective tangent space to $\mathrm{P}^{2}$ at $[X]$ can be identified with the set of lines through $[X]$ in $\mathrm{P}^{2}$. Let $\mathbf{R}^{3 *}$ be another copy of $\mathbf{R}^{3}$, considered as the dual of $\mathbf{R}^{3}$, and $\mathrm{P}^{2 *}$ the projective space on $\mathbf{R}^{3 *}$. If the condition $\langle X, U\rangle=0$ holds for some $X \in \mathbf{R}^{3}-\{0\}$ and $U \in \mathbf{R}^{3 *}-\{0\}$, it holds for all $X^{\prime}$ in the ray through $X$ and $U^{\prime}$ in the ray through $U$, so defines a submanifold $S$ of $\mathrm{P}^{2} \times \mathrm{P}^{2 *}$. Now $[U]$ defines a line in $\mathrm{P}^{2}$ through $[X]$ if and only if $\langle X, U\rangle=0$; so we can identify $S$ with $\mathrm{P}^{2} \mathrm{P}^{2}$. 
But equally, $[X]$ defines a line in $\mathrm{P}^{2 *}$ through $[U]$ if and only if $\langle X, U\rangle=0$, so we can also identify $S$ with $\mathrm{P}^{2} \mathrm{P}^{2 *}$. That is, $S$ with projection on the first factor is $\mathrm{P}^{2} \mathrm{P}^{2}$, with projection on the second factor is $\mathrm{PTP}^{2 *}$. The idea of an element in projective geometry-a point of the manifold $\mathrm{PTP}^{2}$-is self-dual, in the sense that $S$ is invariant under the interchange of $[X]$ and $[U]$.

Let us take coordinates $\left(x, y, y^{\prime}\right)$ on $\mathrm{PTP}^{2}$, such that $(x, y)$ corresponds to $[X]=$ $[x, y, 1] \in \mathrm{P}^{2}$ and $\partial / \partial x+y^{\prime} \partial / \partial y$ is a tangent vector at $[X]$. This vector is tangent to the line $t \mapsto\left[x+t, y+y^{\prime} t, 1\right]$. The 'line coordinates' $[u, v, w]$ of this line must satisfy $u(x+t)+v\left(y+y^{\prime} t\right)+w=0$ for all $\mathrm{t}$; we may take $u=-y^{\prime}, v=1, w=x y^{\prime}-y$, so that $\left(x, y, y^{\prime}\right)$ corresponds to the point

$$
\left([x, y, 1],\left[-y^{\prime}, 1, x y^{\prime}-y\right]\right) \in S .
$$

This gives a way of assigning coordinates to $S$ in which $(x, y)$ are coordinates in the base corresponding to projection on the first factor. Now consider using coordinates for $S$ based on the other projection: we take (for obvious reasons) the base point in the form $[u, 1, w]$, and use coordinates $\left(u, w, w^{\prime}\right)$ such that $\partial / \partial u+w^{\prime} \partial / \partial w$ is the representative tangent vector. Then by a similar argument one finds that the corresponding point in $S$ is

$$
\left(\left[-w^{\prime}, u w^{\prime}-w, 1\right],[u, 1, w]\right)
$$

The coordinate transformation on $S$ relating these two sets of coordinates is $x=-w^{\prime}$, $y=u w^{\prime}-w, y^{\prime}=-u$. Then

$$
\mathrm{d} y-y^{\prime} \mathrm{d} x=u \mathrm{~d} w^{\prime}+w^{\prime} \mathrm{d} u-\mathrm{d} w-u \mathrm{~d} w^{\prime}=-\left(\mathrm{d} w-w^{\prime} \mathrm{d} u\right)
$$

that is to say, the two contact forms, corresponding to the two projections, differ only in sign. Thus the idea of a multiplicity — a vector annihilated by the contact form-is self-dual also.

The plan now is to marry this enhanced structure of the model geometry, associated with the duality of points and lines in two-dimensional projective geometry, with the normal projective connection construction.

Let $M$ and $\bar{M}$ be two two-dimensional manifolds, and $S$ a codimension 1 submanifold of $M \times \bar{M}$, which is fibred over both $M$ and $\bar{M}$ (with one-dimensional fibres). Then for any $\bar{p} \in \bar{M},\{p \in M \mid(p, \bar{p}) \in S\}$ is a path in $M$, say $\sigma_{\bar{p}}$; and for $p \in M,\{\bar{p} \in \bar{M} \mid(p, \bar{p}) \in S\}$ determines a 1-parameter family of paths $\sigma_{\bar{p}} \subset M$ such that $p \in \sigma_{\bar{p}}$ for all such $\bar{p}$. We require that this construction defines a path space on $M$, that is, for every $p \in M$ and $[u] \in \mathrm{P} T_{p} M$ there is a unique $\bar{p} \in \bar{M}$ with $(p, \bar{p}) \in S$ such that the direction of the tangent to $\sigma_{\bar{p}}$ at $x$ is $[u]$. We also require that the similar construction with unbarred and barred quantities interchanged defines a path space on $\bar{M}$. Then we can identify $S \rightarrow M$ with PTM by $(p, \bar{p}) \in S \mapsto[u]$ where $[u]$ is the direction of the tangent to $\sigma_{\bar{p}}$ at $p$; and likewise for $S \rightarrow \bar{M}$ and $\mathrm{P} T \bar{M}$. 
Take coordinates $(x, y)$ on $M$ and $(\bar{x}, \bar{y})$ on $\bar{M}$, and suppose that the submanifold $S$ of $M \times \bar{M}$ is given by $\Phi(x, y, \bar{x}, \bar{y})=0$. Then $\sigma_{\left(\bar{x}_{0}, \bar{y}_{0}\right)}$ is $\Phi\left(x, y, \bar{x}_{0}, \bar{y}_{0}\right)=0$, and the vector

$$
\left(\frac{\partial}{\partial x}+y^{\prime} \frac{\partial}{\partial y}\right)_{\left(x_{0}, y_{0}\right)}
$$

(representative of some tangent vector with $u \neq 0$ ) is tangent to this path if

$$
\Phi_{x}\left(x_{0}, y_{0}, \bar{x}_{0}, \bar{y}_{0}\right)+y^{\prime} \Phi_{y}\left(x_{0}, y_{0}, \bar{x}_{0}, \bar{y}_{0}\right)=0
$$

where $\Phi\left(x_{0}, y_{0}, \bar{x}_{0}, \bar{y}_{0}\right)=0$. Thus the map $S \rightarrow \mathrm{P} T M$ is given by $(x, y, \bar{x}, \bar{y}) \mapsto\left(x, y, y^{\prime}\right)$ where

$$
\Phi(x, y, \bar{x}, \bar{y})=0 \quad \text { and } \quad \Phi_{x}(x, y, \bar{x}, \bar{y})+y^{\prime} \Phi_{y}(x, y, \bar{x}, \bar{y})=0 .
$$

It may appear that $\Phi_{y}$ must be non-zero: but bearing in mind that we have assumed that $u \neq 0$, it becomes clear that we actually require that $\Phi_{x}$ and $\Phi_{y}$ do not vanish simultaneously. Similarly, the map $S \rightarrow \mathrm{P} T \bar{M}$ is given by $(x, y, \bar{x}, \bar{y}) \mapsto\left(\bar{x}, \bar{y}, \bar{y}^{\prime}\right)$ where

$$
\Phi(x, y, \bar{x}, \bar{y})=0 \quad \text { and } \quad \Phi_{\bar{x}}(x, y, \bar{x}, \bar{y})+\bar{y}^{\prime} \Phi_{\bar{y}}(x, y, \bar{x}, \bar{y})=0,
$$

and $\Phi_{\bar{x}}$ and $\Phi_{\bar{y}}$ must not vanish simultaneously.

The condition that the first pair of equations determines $\bar{x}$ and $\bar{y}$ in terms of $x, y$ and $y^{\prime}$ is that the matrix

$$
\left[\begin{array}{ccc}
0 & \Phi_{x} & \Phi_{y} \\
\Phi_{\bar{x}} & \Phi_{x \bar{x}} & \Phi_{y \bar{x}} \\
\Phi_{\bar{y}} & \Phi_{x \bar{y}} & \Phi_{y \bar{y}}
\end{array}\right]
$$

is non-singular; the same condition ensures that the second pair of equations can be solved for $x$ and $y$ in terms of the barred quantities. Notice that this condition subsumes those mentioned immediately above, namely that $\Phi_{x}$ and $\Phi_{y}$ do not vanish simultaneously and neither do $\Phi_{\bar{x}}$ and $\Phi_{\bar{y}}$. We assume therefore that this condition holds everywhere on $S$, and we write it as $\Delta \neq 0$, where $\Delta$ is the determinant,

$$
\begin{aligned}
\Delta & =-\Phi_{x} \Phi_{\bar{x}} \Phi_{y \bar{y}}+\Phi_{x} \Phi_{\bar{y}} \Phi_{y \bar{x}}+\Phi_{y} \Phi_{\bar{x}} \Phi_{x \bar{y}}-\Phi_{y} \Phi_{\bar{y}} \Phi_{x \bar{x}} \\
& =-\Phi_{y} \Phi_{\bar{y}}\left(\Phi_{x \bar{x}}+y^{\prime} \Phi_{y \bar{x}}+\bar{y}^{\prime} \Phi_{x \bar{y}}+y^{\prime} \bar{y}^{\prime} \Phi_{y \bar{y}}\right)
\end{aligned}
$$

for $\Phi_{y} \Phi_{\bar{y}} \neq 0$.

Take some fixed point $(\bar{x}, \bar{y}) \in \bar{M}$ such that the path it defines in $M$ can be parametrized by $x$; then $y^{\prime}=\mathrm{d} y / \mathrm{d} x$, and $\mathrm{d}^{2} y / \mathrm{d} x^{2}$ satisfies

$$
\Phi_{x x}+2 \frac{\mathrm{d} y}{\mathrm{~d} x} \Phi_{x y}+\left(\frac{\mathrm{d} y}{\mathrm{~d} x}\right)^{2} \Phi_{y y}+\frac{\mathrm{d}^{2} y}{\mathrm{~d} x^{2}} \Phi_{y}=0 .
$$


Thus the path is a solution of the second-order differential equation

$$
\frac{\mathrm{d}^{2} y}{\mathrm{~d} x^{2}}=f\left(x, y, \frac{\mathrm{d} y}{\mathrm{~d} x}\right),
$$

where $f\left(x, y, y^{\prime}\right)$ is obtained by eliminating $\bar{x}$ and $\bar{y}$ between the equations

$$
\Phi=0, \quad \Phi_{x}+y^{\prime} \Phi_{y}=0, \quad \Phi_{x x}+2 y^{\prime} \Phi_{x y}+\left(y^{\prime}\right)^{2} \Phi_{y y}+f \Phi_{y}=0 .
$$

The right-hand side $\bar{f}\left(\bar{x}, \bar{y}, \bar{y}^{\prime}\right)$ of the equation giving the dual paths is obtained by eliminating $x$ and $y$ between the equations

$$
\Phi=0, \quad \Phi_{\bar{x}}+\bar{y}^{\prime} \Phi_{\bar{y}}=0, \quad \Phi_{\bar{x} \bar{x}}+2 \bar{y}^{\prime} \Phi_{\bar{x} \bar{y}}+\left(\bar{y}^{\prime}\right)^{2} \Phi_{\bar{y} \bar{y}}+\bar{f} \Phi_{\bar{y}}=0 .
$$

There are two Cartan normal projective connection forms associated with this structure, one corresponding to the differential equation $\mathrm{d}^{2} y / \mathrm{d} x^{2}=f$, the other to the equation $\mathrm{d}^{2} \bar{y} / \mathrm{d} \bar{x}^{2}=\bar{f}$. Each can be represented by a connection form on $S$. The connection associated with the equation $\mathrm{d}^{2} \bar{y} / \mathrm{d} \bar{x}^{2}=\bar{f}$ takes the form

$$
\bar{\omega}=\left[\begin{array}{ccc}
* & * & * \\
\mathrm{~d} \bar{x} & * & * \\
\bar{\theta} & \bar{\phi}-\frac{1}{3} \bar{f}_{\bar{y}^{\prime}} \bar{\theta} & *
\end{array}\right]
$$

with $\bar{\theta}=\mathrm{d} \bar{y}-\bar{y}^{\prime} \mathrm{d} \bar{x}$ and $\bar{\phi}=\mathrm{d} \bar{y}^{\prime}-\bar{f} \mathrm{~d} \bar{x}$. We shall express the lower triangle of $\bar{\omega}$ in terms of unbarred quantities. According to Cartan (see Appendix A), the result should be the anti-transpose of $\omega$, that is, its reflection in the anti-diagonal (which runs from the lower left to the upper right corner). This is not quite the whole story, however: for one thing, we can expect only that it will hold up to a gauge transformation. It will turn out that $\bar{\theta}$ is a scalar multiple of $\theta$; then in view of the effect of a gauge transformation on the lower triangle entries given earlier, it will be enough to work modulo $\theta$.

We must regard $\left(x, y, y^{\prime}\right)$ and $\left(\bar{x}, \bar{y}, \bar{y}^{\prime}\right)$ as alternative coordinates on $S$, with the coordinate transformation given implicitly by

$$
\begin{aligned}
& \Phi(x, y, \bar{x}, \bar{y})=0, \quad \Phi_{x}(x, y, \bar{x}, \bar{y})+y^{\prime} \Phi_{y}(x, y, \bar{x}, \bar{y})=0, \\
& \Phi_{\bar{x}}(x, y, \bar{x}, \bar{y})+\bar{y}^{\prime} \Phi_{\bar{y}}(x, y, \bar{x}, \bar{y})=0,
\end{aligned}
$$

it can be shown that the Jacobian matrix of the variables $\left(\bar{x}, \bar{y}, \bar{y}^{\prime}\right)$ with respect to the variables $\left(x, y, y^{\prime}\right)$ is non-singular by virtue of the assumption that $\Delta \neq 0$. Moreover,

$$
\Phi_{x x}+2 y^{\prime} \Phi_{x y}+\left(y^{\prime}\right)^{2} \Phi_{y y}+f \Phi_{y}=0
$$

and similarly for $\bar{f}$.

In the following working we consider everything as expressed implicitly in terms of the unbarred coordinates. By taking the exterior derivative of the equation $\Phi=0$ and expressing 
$\mathrm{d} y$ and $\mathrm{d} \bar{y}$ in terms of $\theta, \bar{\theta}, \mathrm{d} x$ and $\mathrm{d} \bar{x}$ we obtain

$$
\left(\Phi_{x}+y^{\prime} \Phi_{y}\right) \mathrm{d} x+\Phi_{y} \theta+\left(\Phi_{\bar{x}}+\bar{y}^{\prime} \Phi_{\bar{y}}\right) \mathrm{d} \bar{x}+\Phi_{\bar{y}} \bar{\theta}=0,
$$

so that

$$
\bar{\theta}=-\frac{\Phi_{y}}{\Phi_{\bar{y}}} \theta .
$$

By taking the exterior derivative of the equation $\Phi_{x}+y^{\prime} \Phi_{y}=0$ and expressing all 1-forms in terms of $\mathrm{d} x, \theta, \phi$ and their barred versions we obtain

$$
\begin{aligned}
& \left(\Phi_{x x}+2 y^{\prime} \Phi_{x y}+\left(y^{\prime}\right)^{2} \Phi_{y y}+f \Phi_{y}\right) \mathrm{d} x+\left(\Phi_{x \bar{x}}+y^{\prime} \Phi_{y \bar{x}}+\bar{y}^{\prime} \Phi_{x \bar{y}}+y^{\prime} \bar{y}^{\prime} \Phi_{y \bar{y}}\right) \mathrm{d} \bar{x} \\
& +\left(\Phi_{x y}+y^{\prime} \Phi_{y y}\right) \theta+\left(\Phi_{x \bar{y}}+y^{\prime} \Phi_{y \bar{y}}\right) \bar{\theta}+\Phi_{y} \phi=0 .
\end{aligned}
$$

Thus

$$
\mathrm{d} \bar{x}=\frac{\Phi_{y}^{2} \Phi_{\bar{y}}}{\Delta} \phi \quad(\bmod \theta)
$$

Note that $\Delta$ is unchanged when barred and unbarred quantities are interchanged: thus

$$
\mathrm{d} x=\frac{\Phi_{y} \Phi_{\bar{y}}^{2}}{\Delta} \bar{\phi} \quad(\bmod \theta)
$$

Thus in summary

$$
\mathrm{d} \bar{x}=\frac{\Phi_{y}^{2} \Phi_{\bar{y}}}{\Delta} \phi \quad(\bmod \theta), \quad \bar{\theta}=-\frac{\Phi_{y}}{\Phi_{\bar{y}}} \theta, \quad \bar{\phi}=\frac{\Delta}{\Phi_{y} \Phi_{\bar{y}}^{2}} \mathrm{~d} x \quad(\bmod \theta) .
$$

We now seek by means of a gauge transformation to reduce $\bar{\omega}$ to a form as close as possible to the standard one for a projective connection. Guided by Cartan (see Appendix A), we expect this to involve the interchange of the positions of the $\mathrm{d} x$ and $k \phi+l \theta$ terms, that is to lead to

$$
\left[\begin{array}{ccc}
* & * & * \\
k \phi+l \theta & * & * \\
\theta & \mathrm{d} x & *
\end{array}\right] .
$$

However, in the case of the normal projective connection we will have $k=1$; it is clear from the relations derived above and the formulae for the effects of a gauge transformation derived earlier, that this is impossible, for it would require taking $A, B$ and $C$ (the diagonal 
entries in the gauge transformation matrix) to satisfy

$$
\frac{A}{C}=-\frac{\Phi_{\bar{y}}}{\Phi_{y}}, \quad \frac{A}{B}=\frac{\Delta}{\Phi_{y}^{2} \Phi_{\bar{y}}}, \quad \frac{B}{C}=\frac{\Phi_{y} \Phi_{\bar{y}}^{2}}{\Delta},
$$

and these equations are inconsistent. We cannot prevent a minus sign occurring somewhere in the bottom left-hand corner. For definiteness we take

$$
\frac{A}{C}=\frac{\Phi_{\bar{y}}}{\Phi_{y}}, \quad \frac{A}{B}=\frac{\Delta}{\Phi_{y}^{2} \Phi_{\bar{y}}}, \quad \frac{B}{C}=\frac{\Phi_{y} \Phi_{\bar{y}}^{2}}{\Delta},
$$

the other coefficients of the gauge transformation matrix are chosen so as to eliminate the $\theta$ component of $\bar{\omega}_{2}^{1}$, and also the $\mathrm{d} x$ and $\theta$ components of $\bar{\omega}_{2}^{2}$. That is to say, there is a unique gauge transformation matrix $H$ such that

$$
H^{-1} \bar{\omega} H+H^{-1} \mathrm{~d} H=\left[\begin{array}{ccc}
* & * & * \\
\phi+l \theta & * & * \\
-\theta & \mathrm{d} x & \kappa \phi
\end{array}\right]
$$

We now show that given a trace-free matrix-valued 1-form $\varpi$ with $\varpi^{2}=-\theta, \varpi_{1}^{2}=\mathrm{d} x$, and $\varpi_{2}^{2}$ a multiple of $\phi$, the remaining elements of $\varpi$ are uniquely determined by the following conditions on its curvature form $\Pi$ (defined in the usual way):

- $\Pi$ is strictly upper triangular;

- $\Pi_{2}^{1}$ is a multiple of $\mathrm{d} x \wedge \theta$.

To obtain this result one simply carries out the calculations used to fix the normal projective connection as described in the previous section, but in anti-transposed form, as follows.

The strategy is to compute the relevant components of $\Pi$ in turn, and to examine the consequences of taking them to be zero. In the following calculations $\lambda, \mu$ etc. are functions each of which is arbitrary at the stage at which is introduced, though it is determined subsequently. It is worth noting that for any function $\varphi$,

$$
\mathrm{d} \varphi=\Gamma(\varphi) \mathrm{d} x+\varphi_{y} \theta+\varphi_{y^{\prime}} \phi
$$

Moreover,

$$
\mathrm{d} \theta=-\phi \wedge \mathrm{d} x, \quad \mathrm{~d} \phi=-\mathrm{d} f \wedge \mathrm{d} x=-\left(f_{y} \theta+f_{y^{\prime}} \phi\right) \wedge \mathrm{d} x .
$$

The notation for the components of $\varpi$ and $\Pi$ follows the same system as that for the components of $\omega$. 
First of all

$$
\Pi_{1}^{2}=-\theta \wedge \varpi_{1}^{0}+\mathrm{d} x \wedge\left(\varpi_{1}^{1}-\varpi_{2}^{2}\right)
$$

this vanishes if and only if

$$
\varpi_{1}^{0}=\lambda \mathrm{d} x+\lambda^{\prime} \theta, \quad \varpi_{1}^{1}=\varpi_{2}^{2}+\lambda_{0} \mathrm{~d} x-\lambda \theta .
$$

Next,

$$
\Pi^{2}=-\mathrm{d} \theta-\theta \wedge\left(\varpi_{0}^{0}-\varpi_{2}^{2}\right)+\mathrm{d} x \wedge \varpi^{1}=\left(\phi-\varpi^{1}\right) \wedge \mathrm{d} x-\theta \wedge\left(\varpi_{0}^{0}-\varpi_{2}^{2}\right),
$$

which is zero if and only if the coefficient of $\phi$ in $\varpi^{1}$ is 1 , and

$$
\varpi_{0}^{0}=\varpi_{2}^{2}-\mu \mathrm{d} x+\nu \theta, \quad \varpi^{1}=\phi+\mu \theta .
$$

But the trace of $\varpi$ vanishes, so

$$
0=\left(\lambda_{0}-\mu\right) \mathrm{d} x+(-\lambda+v) \theta+3 \varpi_{2}^{2}=\left(\lambda_{0}-\mu\right) \mathrm{d} x+(-\lambda+v) \theta+3 \kappa \phi,
$$

so that $\kappa=0, \lambda_{0}=\mu, \lambda=v$, and

$$
\varpi_{2}^{2}=0, \quad \varpi_{0}^{0}=-\varpi_{1}^{1}=-\mu \mathrm{d} x+\nu \theta .
$$

Thirdly,

$$
\begin{aligned}
\Pi^{1} & =\mathrm{d}(\phi+\mu \theta)+(\phi+\mu \theta) \wedge\left(\varpi_{0}^{0}-\varpi_{1}^{1}\right)-\varpi_{2}^{1} \wedge \theta \\
& =-\mathrm{d} f \wedge \mathrm{d} x+\mathrm{d} \mu \wedge \theta-\mu \phi \wedge \mathrm{d} x+2(\phi+\mu \theta) \wedge(-\mu \mathrm{d} x+\nu \theta)-\varpi_{2}^{1} \wedge \theta \\
& =\left(3 \mu+f_{y^{\prime}}\right) \mathrm{d} x \wedge \phi+\left(\left(f_{y}+\Gamma(\mu)+2 \mu^{2}\right) \mathrm{d} x+\left(\mu_{y^{\prime}}+2 \nu\right) \phi-\varpi_{2}^{1}\right) \wedge \theta .
\end{aligned}
$$

The necessary and sufficient conditions for $\Pi^{1}=0$ are therefore

$$
\mu=-\frac{1}{3} f_{y^{\prime}}, \quad \varpi_{2}^{1}=\left(f_{y}+\frac{2}{9} f_{y^{\prime}}^{2}-\frac{1}{3} \Gamma\left(f_{y^{\prime}}\right)\right) \mathrm{d} x+\left(2 v-\frac{1}{3} f_{y^{\prime} y^{\prime}}\right) \phi+\rho \theta .
$$

The conditions derived so far are those necessary and sufficient for the connection to be torsionless. We next consider the diagonal elements of $\Pi$.

$$
\Pi_{2}^{2}=-\theta \wedge \varpi_{2}^{0}+\mathrm{d} x \wedge \varpi_{2}^{1}=\left(\varpi_{2}^{0}+\rho \mathrm{d} x\right) \wedge \theta+\left(2 \nu-\frac{1}{3} f_{y^{\prime} y^{\prime}}\right) \mathrm{d} x \wedge \phi
$$

this vanishes if and only if

$$
\varpi_{2}^{0}=-\rho \mathrm{d} x+\sigma \theta, \quad v=\frac{1}{6} f_{y^{\prime} y^{\prime}} .
$$


Notice that the latter condition makes the $\phi$ component of $\varpi_{2}^{1}$ zero. Furthermore,

$$
\begin{aligned}
\Pi_{0}^{0} & =\mathrm{d} \varpi_{0}^{0}+\varpi_{1}^{0} \wedge \varpi^{1}-\varpi_{2}^{0} \wedge \theta \\
& =\mathrm{d}\left(\frac{1}{3} f_{y^{\prime}} \mathrm{d} x+\frac{1}{6} f_{y^{\prime} y^{\prime}} \theta\right)+\left(-\frac{1}{6} f_{y^{\prime} y^{\prime}} \mathrm{d} x+\lambda^{\prime} \theta\right) \wedge\left(\phi-\frac{1}{3} f_{y^{\prime}} \theta\right)+\rho \mathrm{d} x \wedge \theta \\
& =\left(\rho-\frac{1}{3} f_{y y^{\prime}}-\frac{1}{18} f_{y^{\prime}} f_{y^{\prime} y^{\prime}}+\frac{1}{6} \Gamma\left(f_{y^{\prime} y^{\prime}}\right)\right) \mathrm{d} x \wedge \theta+\left(\lambda^{\prime}-\frac{1}{6} f_{y^{\prime} y^{\prime} y^{\prime}}\right) \theta \wedge \phi .
\end{aligned}
$$

So we require that

$$
\rho=\frac{1}{3} f_{y y^{\prime}}+\frac{1}{18} f_{y^{\prime}} f_{y^{\prime} y^{\prime}}-\frac{1}{6} \Gamma\left(f_{y^{\prime} y^{\prime}}\right), \quad \lambda^{\prime}=\frac{1}{6} f_{y^{\prime} y^{\prime} y^{\prime}}
$$

for $\Pi_{0}^{0}$ to be zero. When this holds, $\Pi_{1}^{1}$ will be zero also, since the trace of $\Pi$ must vanish.

We have now fixed the whole of $\varpi$ with the exception of the coefficient $\sigma$ in $\varpi_{2}^{0}$. We determine this by imposing the condition that $\Pi_{2}^{1}$ be semi-basic, that is, that it be a multiple of $\mathrm{d} x \wedge \theta$. Before proceeding to evaluate $\Pi_{2}^{1}$ it is useful to note that

$$
\frac{\partial}{\partial y^{\prime}}\left(f_{y}+\frac{2}{9} f_{y^{\prime}}^{2}-\frac{1}{3} \Gamma\left(f_{y^{\prime}}\right)\right)=\frac{2}{3} f_{y y^{\prime}}+\frac{1}{9} f_{y^{\prime}} f_{y^{\prime} y^{\prime}}-\frac{1}{3} \Gamma\left(f_{y^{\prime} y^{\prime}}\right)=2 \rho .
$$

Using this we find that

$$
\left.\frac{\partial}{\partial y^{\prime}}\right\lrcorner \Pi_{2}^{1}=\left(\rho_{y^{\prime}}+\sigma\right) \theta
$$

so $\Pi_{2}^{1}$ will be semi-basic if and only if $\sigma=-\rho_{y^{\prime}}$.

This completes the determination of $\varpi$. We see that in fact $\varpi$ is given in terms of the first normal projective connection $\omega$ by

$$
\begin{aligned}
& \varpi_{0}^{0}=-\omega_{2}^{2}, \quad \varpi_{1}^{0}=\omega_{2}^{1}, \quad \varpi_{2}^{0}=-\omega_{2}^{0}, \quad \varpi^{1}=\omega_{1}^{2}, \quad \varpi_{1}^{1}=-\omega_{1}^{1}, \\
& \varpi_{2}^{1}=\omega_{1}^{0}, \quad \varpi^{2}=-\omega^{2}, \quad \varpi_{1}^{2}=\omega^{1}, \quad \varpi_{2}^{2}=-\omega_{0}^{0} .
\end{aligned}
$$

Note the differences in sign from Cartan's version given in Appendix A. In fact

$$
\varpi=-K \omega^{\mathrm{T}} K
$$

where $\omega^{\mathrm{T}}$ is the transpose of $\omega$ and $K$ is the matrix

$$
K=\left[\begin{array}{ccc}
0 & 0 & -1 \\
0 & 1 & 0 \\
-1 & 0 & 0
\end{array}\right]
$$

It is easy to check that when $\varpi$ and $\omega$ are related as above, their curvatures $\Pi$ and $\Omega$ are related in the same way: this would not be true if the minus signs did not appear in the relationship, and indeed there would be no obvious relation between the curvature 
components if that were the case. The essential point is that $M \mapsto-K M^{\mathrm{T}} K=M^{\prime}$ is a homomorphism of the matrix Lie algebra:

$$
\left[M_{1}^{\prime}, M_{2}^{\prime}\right]=K\left[M_{1}^{\mathrm{T}}, M_{2}^{\mathrm{T}}\right] K=-K\left[M_{1}, M_{2}\right]^{\mathrm{T}} K=\left[M_{1}, M_{2}\right]^{\prime} .
$$

Indeed, this is true for any $K$ for which $K^{2}$ is the identity; but without the overall minus sign one obtains instead an anti-homomorphism, and then

$$
\mathrm{d} \omega^{\prime}+\frac{1}{2}\left[\omega^{\prime} \wedge \omega^{\prime}\right]=\mathrm{d} \omega^{\prime}-\frac{1}{2}[\omega \wedge \omega]^{\prime}
$$

It follows from the fact that $\Pi=-K \Omega^{\mathrm{T}} K$ that

$$
\Pi=\left[\begin{array}{ccc}
0 & a \theta \wedge \phi & * \\
0 & 0 & b \mathrm{~d} x \wedge \theta \\
0 & 0 & 0
\end{array}\right]
$$

Consider again the gauged version of the second normal projective connection, $H^{-1} \bar{\omega} H+$ $H^{-1} \mathrm{~d} H$. Its curvature is $H^{-1} \bar{\Omega} H$, where

$$
\bar{\Omega}=\left[\begin{array}{ccc}
0 & \bar{b} \mathrm{~d} \bar{x} \wedge \bar{\theta} & * \\
0 & 0 & \bar{a} \bar{\theta} \wedge \bar{\phi} \\
0 & 0 & 0
\end{array}\right]
$$

That is,

$$
H^{-1} \bar{\Omega} H=\left[\begin{array}{ccc}
0 & A^{-1} B \bar{b} \mathrm{~d} \bar{x} \wedge \bar{\theta} & * \\
0 & 0 & B^{-1} C \bar{a} \bar{\theta} \wedge \bar{\phi} \\
0 & 0 & 0
\end{array}\right]=\left[\begin{array}{ccc}
0 & \beta \theta \wedge \phi & * \\
0 & 0 & \alpha \mathrm{d} x \wedge \theta \\
0 & 0 & 0
\end{array}\right]
$$

where

$$
\alpha=\frac{\Delta}{\left(\Phi_{\bar{y}}\right)^{3}} \frac{C}{B} \bar{a}=\frac{\Delta^{2}}{\Phi_{y}\left(\Phi_{\bar{y}}\right)^{5}} \bar{a},
$$

and

$$
\beta=\frac{\left(\Phi_{y}\right)^{3}}{\Delta} \frac{B}{A} \bar{b}=\frac{\left(\Phi_{y}\right)^{5} \Phi_{\bar{y}}}{\Delta^{2}} \bar{b} .
$$

Thus $H^{-1} \bar{\omega} H+H^{-1} \mathrm{~d} H$ satisfies the conditions that uniquely determine $\varpi$, and therefore is $\varpi$ :

$$
H^{-1} \bar{\omega} H+H^{-1} \mathrm{~d} H=\varpi .
$$


It follows that $H^{-1} \bar{\Omega} H=\Pi$, so the coefficients $\alpha$ and $\beta$ are just $b$ and $a$, respectively, whence

$$
\bar{a}=\frac{\Phi_{y}\left(\Phi_{\bar{y}}\right)^{5}}{\Delta^{2}} b, \quad \bar{b}=\frac{\Delta^{2}}{\left(\Phi_{y}\right)^{5} \Phi_{\bar{y}}} a .
$$

Thus the vanishing of $b$ is the necessary and sufficient condition for the dual secondorder differential equation to be projectively affine; moreover, if a second-order differential equation and its dual are both projectively affine then the first equation is rectifiable, and of course its dual is rectifiable also. This interpretation of the significance of $b$ is not drawn explicitly by Cartan, though it is implicit in what he writes. It has been derived recently in [7] and [8], by methods differing from each other and from Cartan's.

There is a further fact worth pointing out. Cartan says, in relation to the coefficients of the curvature of the normal projective connection, that

$$
\begin{aligned}
& \int \sqrt[4]{a b} \omega^{2}, \quad \iiint \sqrt{a b} \omega^{1} \omega^{2} \omega_{1}^{2}, \quad \iint a^{1 / 8} b^{5 / 8} \omega^{1} \omega^{2}, \\
& \iint a^{5 / 8} b^{1 / 8} \omega^{2} \omega_{1}^{2}
\end{aligned}
$$

are 'invariant integrals'; that is to say,

$$
\sqrt[4]{a b} \theta, \quad \sqrt{a b} \mathrm{~d} x \wedge \theta \wedge \phi, \quad a^{1 / 8} b^{5 / 8} \mathrm{~d} x \wedge \theta, \quad a^{5 / 8} b^{1 / 8} \theta \wedge \phi
$$

are well-defined forms. These forms are essentially invariant under duality; in fact

$$
\sqrt[4]{\bar{a} \bar{b}} \bar{\theta}=-\sqrt[4]{a b} \theta, \quad \sqrt{\bar{a} \bar{b}} \mathrm{~d} \bar{x} \wedge \bar{\theta} \wedge \bar{\phi}=-\sqrt{a b} \mathrm{~d} x \wedge \theta \wedge \phi
$$

while

$$
\bar{a}^{1 / 8} \bar{b}^{5 / 8} \mathrm{~d} \bar{x} \wedge \bar{\theta}=a^{5 / 8} b^{1 / 8} \theta \wedge \phi, \quad \bar{a}^{5 / 8} \bar{b}^{1 / 8} \bar{\theta} \wedge \bar{\phi}=a^{1 / 8} b^{5 / 8} \mathrm{~d} x \wedge \theta .
$$

\section{Contact structure and duality}

The condition $\Delta \neq 0$ imposed in the last section may be interpreted in another way, pointed out in [2]: it states that the 1-form $\vartheta=\Phi_{x} \mathrm{~d} x+\Phi_{y} \mathrm{~d} y=-\left(\Phi_{\bar{x}} \mathrm{~d} \bar{x}+\Phi_{\bar{y}} \mathrm{~d} \bar{y}\right)$ satisfies $\vartheta \wedge \mathrm{d} \vartheta \neq 0$ on $S$ and therefore defines a contact structure on this three-dimensional manifold. From the point of view of the last section this is not at all surprising, since $\vartheta$ is a multiple of $\mathrm{d} y-y^{\prime} \mathrm{d} x$, which is a contact form and is even in standard coordinate representation. However, this observation suggests approaching the question in a different way, and when one does so the duality of the connection becomes somewhat more transparent.

Suppose given a three-dimensional manifold $S$ equipped with a contact structure, which it will be convenient to think of as a two-dimensional distribution $\mathcal{D}$ which is non-integrable 
in the sense that for any pair of linearly independent vector fields $X$ and $Y$ in $\mathcal{D},[X, Y] \notin \mathcal{D}$. Any 1-form $\vartheta$ on $S$ which is an annihilator of $\mathcal{D}$ satisfies the condition $\vartheta \wedge \mathrm{d} \vartheta \neq 0$. We further suppose that a basis has been chosen for $\mathcal{D}$, and we denote the basis vectors by $X$ and $\bar{X}$. Then $\{X, \bar{X},[X, \bar{X}]\}$ is a basis for vector fields on $S$; let $\{\varphi, \bar{\varphi}, \vartheta\}$ be the dual basis of 1 -forms.

In the case under consideration in the previous section we would take $X$ to be tangent to one of the fibres of the double fibration of $S$ and $\bar{X}$ to the other; $\mathcal{D}$ would be the distribution spanned by $X$ and $\bar{X}$ and $\vartheta$ would be a scalar multiple of $\Phi_{x} \mathrm{~d} x+\Phi_{y} \mathrm{~d} y$. The purpose of the present discussion is to examine again the effect on the Cartan connection form of interchanging the roles of the fibrations, but in doing so to treat them on an equal footing. We will accomplish this by working in terms of the dual bases described above; when we interchange $X$ and $\bar{X}$ the new 1-form basis becomes $\{\bar{\varphi}, \varphi,-\vartheta\}$. For the normal Cartan projective connection described in previous sections we have

$$
X=\Gamma, \quad \bar{X}=\frac{\partial}{\partial y^{\prime}},
$$

and therefore

$$
[X, \bar{X}]=-\frac{\partial}{\partial y}-f_{y^{\prime}} \frac{\partial}{\partial y^{\prime}}
$$

the 1 -forms $\mathrm{d} x, \phi-(1 / 3) f_{y^{\prime}} \theta, \theta$ which occupy the lower triangle positions in the connection matrix are not dual to this basis of vector fields, and herein lies the main difference between the previous discussion and the following one. The dual 1-form basis is actually $\{\mathrm{d} x, \phi-$ $f_{y^{\prime}} \theta,-\theta$ \}. In discussing the Cartan connection from the new point of view we will therefore take the lower triangle of the connection form to be

$$
\omega=\left[\begin{array}{ccc}
* & * & * \\
\varphi & * & * \\
-\vartheta & \bar{\varphi} & *
\end{array}\right]
$$

in the case just discussed this will be gauge-equivalent to the version used previously. We emphasise that now $X$ may be any vector field tangent to the first fibration and $\bar{X}$ any vector field tangent to the second. Thus in the present version of the theory transformations of the form $X \mapsto \lambda X, \bar{X} \mapsto \bar{\lambda} \bar{X}$, for any non-vanishing functions $\lambda$ and $\bar{\lambda}$, will be allowed; such transformations induce gauge transformations of the kind discussed previously, with coefficients given by $\lambda, \bar{\lambda}$ and their derivatives. Little more need be said about this point.

It follows from their definitions that the exterior derivatives of the basis 1-forms can be written as

$$
\mathrm{d} \varphi=\psi \wedge \vartheta, \quad \mathrm{d} \bar{\varphi}=-\bar{\psi} \wedge \vartheta, \quad \mathrm{d} \vartheta=-\varphi \wedge \bar{\varphi}+\chi \wedge \vartheta
$$

where $\psi, \bar{\psi}$ and $\chi$ are certain 1 -forms which are linear combinations of $\varphi$ and $\bar{\varphi}$. 
It will be convenient to change notation: we will denote the connection form by

$$
\omega=\left[\begin{array}{ccc}
\alpha & \beta & \gamma \\
\varphi & -\left(\alpha+\alpha^{\prime}\right) & \beta^{\prime} \\
-\vartheta & \bar{\varphi} & \alpha^{\prime}
\end{array}\right]
$$

We are assuming that $X$ and $\bar{X}$, and therefore $\varphi, \bar{\varphi}$ and $\vartheta$, are fixed; thus the only remaining gauge freedom is that coming from a gauge transformation of the form

$$
H=\left[\begin{array}{lll}
1 & 0 & F \\
0 & 1 & 0 \\
0 & 0 & 1
\end{array}\right]
$$

for which

$$
H^{-1} \omega H+H^{-1} \mathrm{~d} H=\left[\begin{array}{ccc}
\alpha+F \vartheta & * & * \\
\varphi & -\left(\alpha+\alpha^{\prime}\right) & * \\
-\vartheta & \bar{\varphi} & \alpha^{\prime}-F \vartheta
\end{array}\right]
$$

We may therefore fix the gauge by requiring that $\alpha-\alpha^{\prime}$ is independent of $\vartheta$ (that is, that it is a linear combination of $\varphi$ and $\bar{\varphi}$ ), and this we do henceforth.

It is not difficult to show, using the same kind of argument as the one in the previous section, that $\alpha, \alpha^{\prime}, \beta, \beta^{\prime}$ and $\gamma$ are uniquely determined, in terms of $\psi, \bar{\psi}$ and $\chi$ and their derivatives, by the requirements that the curvature $\Omega$ of $\omega$ takes the form

$$
\Omega=\left[\begin{array}{lll}
0 & B & * \\
0 & 0 & B^{\prime} \\
0 & 0 & 0
\end{array}\right]
$$

with $B$ a multiple of $\varphi \wedge \vartheta$. The conditions that the torsion vanishes amount to the following equations:

$$
\begin{aligned}
& \mathrm{d} \varphi-\left(2 \alpha+\alpha^{\prime}\right) \wedge \varphi-\beta^{\prime} \wedge \vartheta=0, \quad \mathrm{~d} \bar{\varphi}+\left(\alpha+2 \alpha^{\prime}\right) \wedge \bar{\varphi}+\beta \wedge \vartheta=0, \\
& \mathrm{~d} \vartheta-\left(\alpha-\alpha^{\prime}\right) \wedge \vartheta+\varphi \wedge \bar{\varphi}=0 .
\end{aligned}
$$

It follows from the last of these, together with the gauge-fixing assumption, that $\alpha-\alpha^{\prime}=\chi$. The first two equations then determine the $\varphi$ and $\bar{\varphi}$ components of $\alpha$, and therefore $\alpha^{\prime}$, in terms of $\psi, \bar{\psi}$ and $\chi$, and the $\varphi$ and $\bar{\varphi}$ components of $\beta$ and $\beta^{\prime}$ in terms of $\alpha$ and $\alpha^{\prime}$. The conditions that the diagonal elements of $\Omega$ vanish are

$$
\mathrm{d} \alpha+\beta \wedge \varphi-\gamma \wedge \vartheta=0, \quad \mathrm{~d} \alpha^{\prime}-\beta^{\prime} \wedge \bar{\varphi}+\gamma \wedge \vartheta=0,
$$


or equivalently

$$
\mathrm{d}\left(\alpha+\alpha^{\prime}\right)+\beta \wedge \varphi-\beta^{\prime} \wedge \bar{\varphi}=0, \quad \mathrm{~d}\left(\alpha-\alpha^{\prime}\right)+\beta \wedge \varphi+\beta^{\prime} \wedge \bar{\varphi}=2 \gamma \wedge \vartheta .
$$

The $\varphi \wedge \bar{\varphi}$ component of the first of these determines the $\vartheta$ component of $\alpha+\alpha^{\prime}$, and therefore of $\alpha$ and $\alpha^{\prime}$ since they have the same $\vartheta$ component; the remaining components determine the $\vartheta$ components of $\beta$ and $\beta^{\prime}$. The $\varphi \wedge \bar{\varphi}$ component of the second equation is satisfied identically, and the other two components give the $\varphi$ and $\bar{\varphi}$ components of $\gamma$. It remains to find the $\vartheta$ component of $\gamma$. We have

$$
B=\mathrm{d} \beta+\left(2 \alpha+\alpha^{\prime}\right) \wedge \beta+\gamma \wedge \bar{\varphi}, \quad B^{\prime}=\mathrm{d} \beta^{\prime}-\left(\alpha+2 \alpha^{\prime}\right) \wedge \beta^{\prime}-\gamma \wedge \varphi .
$$

We show first that $B \wedge \vartheta=0$, from which it follows that $B$ is a multiple of $\varphi \wedge \vartheta$ if and only if $B \wedge \varphi=0$, a condition which clearly determines the $\vartheta$ component of $\gamma$. Now by taking the exterior derivative of the second of the torsion equations we find that

$$
\begin{aligned}
\mathrm{d} \beta \wedge \vartheta & =\beta \wedge \mathrm{d} \vartheta-\mathrm{d}\left(\alpha+2 \alpha^{\prime}\right) \wedge \bar{\varphi}+\left(\alpha+2 \alpha^{\prime}\right) \wedge \mathrm{d} \bar{\varphi} \\
& =\beta \wedge \mathrm{d} \vartheta+\beta \wedge \varphi \wedge \bar{\varphi}+\gamma \wedge \vartheta \wedge \bar{\varphi}-\left(\alpha+2 \alpha^{\prime}\right) \wedge \beta \wedge \vartheta \\
& =\beta \wedge\left(\mathrm{d} \vartheta+\varphi \wedge \bar{\varphi}+\left(\alpha+2 \alpha^{\prime}\right) \wedge \vartheta\right)-\gamma \wedge \bar{\varphi} \wedge \vartheta \\
& =\left(-\left(2 \alpha+\alpha^{\prime}\right) \wedge \beta-\gamma \wedge \varphi\right) \wedge \vartheta,
\end{aligned}
$$

which is to say that $B \wedge \vartheta=0$. A similar argument shows that $B^{\prime} \wedge \vartheta=0$.

Thus $B$ will be a multiple of $\varphi \wedge \vartheta$ if and only if

$$
\gamma \wedge \varphi \wedge \bar{\varphi}=\left(\mathrm{d} \beta+\left(2 \alpha+\alpha^{\prime}\right) \wedge \beta\right) \wedge \varphi
$$

But

$$
\mathrm{d} \beta \wedge \varphi=\beta \wedge \mathrm{d} \varphi+\mathrm{d}(\gamma \wedge \vartheta)
$$

from which it follows that

$$
\left(\mathrm{d} \beta+\left(2 \alpha+\alpha^{\prime}\right) \wedge \beta\right) \wedge \varphi=\beta \wedge \beta^{\prime} \wedge \vartheta+\mathrm{d}(\gamma \wedge \vartheta)
$$

A very similar calculation gives

$$
\left(\mathrm{d} \beta^{\prime}-\left(\alpha+2 \alpha^{\prime}\right) \wedge \beta^{\prime}\right) \wedge \bar{\varphi}=\beta \wedge \beta^{\prime} \wedge \vartheta+\mathrm{d}(\gamma \wedge \vartheta)=\left(\mathrm{d} \beta+\left(2 \alpha+\alpha^{\prime}\right) \wedge \beta\right) \wedge \varphi .
$$

That is to say, $B \wedge \varphi=0$ if and only if $B^{\prime} \wedge \bar{\varphi}=0$, so that the conditions that $B$ is a multiple of $\varphi \wedge \vartheta$ and $B^{\prime}$ is a multiple of $\bar{\varphi} \wedge \vartheta$ are the same.

We can now discuss the effects of the duality transformation $X \mapsto \bar{X}, \bar{X} \mapsto X$. The notation is supposed to suggest the idea that this acts like complex conjugation; we should 
then think of $\vartheta$ as purely imaginary, and $\chi$ as real. The dual connection form $\bar{\omega}$,

$$
\bar{\omega}=\left[\begin{array}{ccc}
\bar{\alpha} & \bar{\beta} & \bar{\gamma} \\
\bar{\varphi} & -\left(\bar{\alpha}+\bar{\alpha}^{\prime}\right) & \bar{\beta}^{\prime} \\
\vartheta & \varphi & \bar{\alpha}^{\prime}
\end{array}\right],
$$

is assumed to be gauged so that $\bar{\alpha}-\bar{\alpha}^{\prime}$ is independent of $\vartheta$, as before. The connection is then uniquely determined by the conditions

$$
\begin{aligned}
& \mathrm{d} \bar{\varphi}-\left(2 \bar{\alpha}+\bar{\alpha}^{\prime}\right) \wedge \bar{\varphi}+\bar{\beta}^{\prime} \wedge \vartheta=0, \quad \mathrm{~d} \varphi+\left(\bar{\alpha}+2 \bar{\alpha}^{\prime}\right) \wedge \varphi-\bar{\beta} \wedge \vartheta=0, \\
& \mathrm{~d} \vartheta-\left(\bar{\alpha}-\bar{\alpha}^{\prime}\right) \wedge \vartheta+\varphi \wedge \bar{\varphi}=0
\end{aligned}
$$

(vanishing of torsion),

$$
\mathrm{d} \bar{\alpha}+\bar{\beta} \wedge \bar{\varphi}+\bar{\gamma} \wedge \vartheta=0, \quad \mathrm{~d} \bar{\alpha}^{\prime}-\bar{\beta}^{\prime} \wedge \varphi-\bar{\gamma} \wedge \vartheta=0
$$

(vanishing of the diagonal elements of $\bar{\Omega}$ ); and

$$
\bar{\gamma} \wedge \varphi \wedge \bar{\varphi}=-\left(\mathrm{d} \bar{\beta}+\left(2 \bar{\alpha}+\bar{\alpha}^{\prime}\right) \wedge \bar{\beta}\right) \wedge \bar{\varphi}=-\left(\mathrm{d} \bar{\beta}^{\prime}-\left(\bar{\alpha}+2 \bar{\alpha}^{\prime}\right) \wedge \bar{\beta}^{\prime}\right) \wedge \varphi
$$

$\left(\bar{B} \wedge \bar{\varphi}=0\right.$ or equivalently $\left.\bar{B}^{\prime} \wedge \varphi=0\right)$.

From the torsion equations (both the initial ones and their conjugates) we obtain, first, $\bar{\alpha}-$ $\bar{\alpha}^{\prime}=\alpha-\alpha^{\prime}=\chi$, whence $\alpha^{\prime}+\bar{\alpha}=\alpha+\bar{\alpha}^{\prime}$. Using this in the other two pairs of equations, which give

$$
\begin{aligned}
& \left(2\left(\alpha+\bar{\alpha}^{\prime}\right)+\left(\alpha^{\prime}+\bar{\alpha}\right) \wedge \varphi+\left(\beta^{\prime}-\bar{\beta}\right)\right) \wedge \vartheta=0, \\
& \left(\left(\alpha+\bar{\alpha}^{\prime}\right)+2\left(\alpha^{\prime}+\bar{\alpha}\right) \wedge \bar{\varphi}+\left(\beta-\bar{\beta}^{\prime}\right)\right) \wedge \vartheta=0,
\end{aligned}
$$

we find that

$$
\left(\alpha^{\prime}+\bar{\alpha}\right) \wedge \varphi \wedge \vartheta=\left(\alpha^{\prime}+\bar{\alpha}\right) \wedge \bar{\varphi} \wedge \vartheta=0
$$

Thus $\alpha^{\prime}+\bar{\alpha}$ is a multiple of $\vartheta$, say $\kappa \vartheta$, and likewise $\alpha+\bar{\alpha}^{\prime}=\kappa \vartheta$. But then $\left(\beta^{\prime}-\bar{\beta}\right) \wedge \vartheta=$ $-3 \kappa \vartheta \wedge \varphi$. On the other hand,

$$
\mathrm{d}\left(\alpha^{\prime}+\bar{\alpha}\right)=\mathrm{d} \kappa \wedge \vartheta+\kappa(\varphi \wedge \bar{\varphi}-\chi \wedge \vartheta)=\left(\beta^{\prime}-\bar{\beta}\right) \wedge \bar{\varphi}-(\gamma+\bar{\gamma}) \wedge \vartheta
$$

taking the exterior product with $\vartheta$ gives $\kappa=0$. Thus $\alpha^{\prime}=-\bar{\alpha}$ and $\bar{\alpha}^{\prime}=-\alpha$. Moreover, $\beta^{\prime}-\bar{\beta}$ is a multiple of $\vartheta$, and so similarly is $\beta-\bar{\beta}^{\prime}$; but

$$
\left(\beta^{\prime}-\bar{\beta}\right) \wedge \bar{\varphi}=(\gamma+\bar{\gamma}) \wedge \vartheta=\left(\beta-\bar{\beta}^{\prime}\right) \wedge \varphi
$$

whence $\beta^{\prime}=\bar{\beta}, \bar{\beta}^{\prime}=\beta$, and $\gamma \wedge \vartheta=-\bar{\gamma} \wedge \vartheta$. Finally, from the conditions on $B$ and $\bar{B}$,

$$
\bar{\gamma} \wedge \varphi \wedge \bar{\varphi}=-\left(\mathrm{d} \beta+\left(\alpha^{\prime}+2 \alpha\right) \wedge \beta\right) \wedge \varphi=-\gamma \wedge \varphi \wedge \bar{\varphi}
$$


so $\bar{\gamma}=-\gamma$.

It follows that we can write

$$
\omega=\left[\begin{array}{ccc}
\alpha & \beta & \gamma \\
\varphi & -(\alpha-\bar{\alpha}) & \bar{\beta} \\
-\vartheta & \bar{\varphi} & -\bar{\alpha}
\end{array}\right]
$$

with $\bar{\gamma}=-\gamma$; and $\bar{\omega}=-K \omega^{\mathrm{T}} K$, as explained in the previous section. Thus $\bar{\Omega}=-K \Omega^{\mathrm{T}} K$, and so $B^{\prime}=\bar{B}, \bar{B}^{\prime}=B$.

\section{CR structures}

It is mentioned in [2] and shown in detail in [9] that there is a close analogy between the Cartan geometry of second-order differential equations, on the one hand, and the geometry of three-dimensional Cauchy-Riemann structures, or CR structures, on the other. The methods of the previous section make this analogy particularly obvious.

The geometry of CR structures deals with codimension 1 real submanifolds of $\mathbf{C}^{2}$, and is concerned with finding invariants of such submanifolds with respect to biholomorphic transformations of $\mathbf{C}^{2}$. We can define such a submanifold, say $S$, as the zero set of a suitable real-valued function $\Phi(x, y, \bar{x}, \bar{y})$ on $\mathbf{C}^{2}$, where now $x$ and $y$ are complex coordinates, and the bar really does mean the complex conjugate. Since

$$
\Phi_{x} \mathrm{~d} x+\Phi_{y} \mathrm{~d} y+\Phi_{\bar{x}} \mathrm{~d} \bar{x}+\Phi_{\bar{y}} \mathrm{~d} \bar{y}=0
$$

on $S$, if we set $\vartheta=\Phi_{x} \mathrm{~d} x+\Phi_{y} \mathrm{~d} y=-\left(\Phi_{\bar{x}} \mathrm{~d} \bar{x}+\Phi_{\bar{y}} \mathrm{~d} \bar{y}\right)$ then $\vartheta$ is pure imaginary. The complex vector fields $X$ and $\bar{X}$, where

$$
X=\Phi_{y} \frac{\partial}{\partial x}-\Phi_{x} \frac{\partial}{\partial y}
$$

and $\bar{X}$ is its conjugate span the two-dimensional distribution annihilated by $\vartheta$; under a biholomorphic transformation of $\mathbf{C}^{2}, X$ and $\bar{X}$ get multiplied by complex scalars. If the bracket $[X, \bar{X}]$ is everywhere linearly independent of $X$ and $\bar{X}$, or equivalently if $\vartheta \wedge d \vartheta \neq 0$, then the CR structure is said to be non-degenerate (see [9]); by scaling as necessary we can then choose a complex 1 -form basis $\{\varphi, \bar{\varphi}, \vartheta\}$ adapted to the structure, with $\bar{\vartheta}=-\vartheta$.

The arguments of the previous section may now be repeated more-or-less verbatim to obtain the following result: for given $X$ there is a unique $\mathfrak{s l}(3, \mathbf{C})$-valued 1-form $\omega$ on $S$ with

$$
\omega=\left[\begin{array}{ccc}
\alpha & * & * \\
\varphi & * & * \\
-\vartheta & \bar{\varphi} & \alpha^{\prime}
\end{array}\right],
$$


$\alpha-\alpha^{\prime}$ being independent of $\vartheta$, whose curvature $\Omega$ is strictly upper triangular,

$$
\Omega=\left[\begin{array}{ccc}
0 & B & * \\
0 & 0 & B^{\prime} \\
0 & 0 & 0
\end{array}\right]
$$

where $B$ is a multiple of $\varphi \wedge \vartheta$. In fact

$$
\omega=\left[\begin{array}{ccc}
\alpha & \beta & \gamma \\
\varphi & -(\alpha-\bar{\alpha}) & \bar{\beta} \\
-\vartheta & \bar{\varphi} & -\bar{\alpha}
\end{array}\right]
$$

for certain complex 1 -forms $\alpha, \beta$ and $\gamma$, where $\gamma$ is pure imaginary. Moreover $B^{\prime}=\bar{B}$, and $B$ is a relative invariant of the structure. Furthermore, the conjugate of $\omega$ is given by $\bar{\omega}=-K \omega^{\mathrm{T}} K$, or equivalently

$$
\omega K+K \omega^{\dagger}=0, \quad \omega^{\dagger}=\bar{\omega}^{\mathrm{T}} .
$$

Now $K$ defines a Hermitian form of signature $(++-)$, so we see that $\omega$ takes its values in $\mathfrak{s u}(2,1)$. This reproduces, albeit in gauged form, and with some minor differences in numerical factors, the basic analysis of [9].

\section{Acknowledgements}

The first author is a Guest Professor at Ghent University, and a Visiting Senior Research Fellow of King's College, University of London: he is grateful to the Department of Mathematical Physics and Astronomy at Ghent and the Department of Mathematics at King's for their hospitality. Thanks are due also to Pawel Nurowski for several enlightening conversations.

\section{Appendix A. What Cartan says about duality}

Here is the relevant section, 23, of Cartan's paper (with the notation brought up to date where necessary):

La notion d'élément est à elle-même, en géométrie projective, sa propre dualistique, ainsi que la notion de multiplicité. Par suite toute variété d'éléments à connexion projective se transforme par dualité en une autre variété d'éléments à connexion projective, les points de la première correspondant aux géodésiques de la seconde, et réciproquement. Si l'on désigne par la lettre $\varpi$ les composantes de la connexion projective de la seconde, on a, comme il est facile de voir,

$$
\begin{aligned}
& \varpi_{0}^{0}=\omega_{2}^{2}, \quad \varpi^{1}=\omega_{1}^{2}, \quad \varpi^{2}=\omega^{2}, \quad \varpi_{1}^{0}=\omega_{2}^{1}, \quad \varpi_{1}^{1}=\omega_{1}^{1}, \\
& \varpi_{1}^{2}=\omega^{1}, \quad \varpi_{2}^{0}=\omega_{2}^{0}, \quad \varpi_{2}^{1}=\omega_{1}^{0}, \quad \varpi_{2}^{2}=\omega_{0}^{0} \text {. }
\end{aligned}
$$


Les relations qui appartiennent à une connexion projective normale se transforment donc par dualité en

$$
\Pi^{2}=0, \quad \Pi_{1}^{2}=0, \quad \Pi^{1}=0, \quad \Pi_{1}^{1}-\Pi_{2}^{2}=0, \quad \Pi_{0}^{0}-\Pi_{2}^{2}=0 ;
$$

elles conservent la même forme. Quant à la condition que le coefficient $r \operatorname{de} \omega^{1} \wedge \omega^{2}$ dans $\Omega_{2}^{1}$ est nul, elle devient la condition que le coefficient de $\varpi^{2} \wedge \varpi_{1}^{2}$ dans $\Pi_{1}^{0}$ soit nul. Autrement dit, la dualistique d'une variété d'éléments à connexion projective normale est encore une variété d'éléments à connexion projective normale.

La relation qui existe entre les deaux familles de géodésiques de deux variétés normales dualistes est évidente. Si

$$
F(x, y, a, b)=0
$$

est l'équation générale des géodésiques de la première, lorsqu'on y regarde $x$ et $y$ comme les variables ponctuelles et $a, b$ comme les constantes arbitraires, c'est aussi l'équation des géodésiques de la seconde variété, à condition d'y regarder $a$ et $b$ comme les variables ponctuelles, $x$ et $y$ comme les constantes arbitraires. La relation entre les variétés normales dualistes se traduit donc analytiquement par une certaine correspondance entre deux équations différentielles ordinaires du seconde ordre (ou plutôt entre les deux classes d'équations différentielles qu'on obtient en transformant chacune d'elles par une transformation ponctuelle arbitraire). Cette correspondence a déjà été étudiée par M.A. Koppisch sous son aspect purement analytique.

\section{References}

[1] L. Berwald, Ueber systeme von gewöhnlichen differentialgleichungen zweiter ordnung, deren integralkurven mit dem system der geraden linien topologisch äquivalent sind, Ann. Math. 48 (1947) 193-215.

[2] R.L. Bryant, Élie Cartan and geometric duality, Journées Élie Cartan 1998 \& 1999: Inst. É. Cartan 16 (2000) 5-20.

[3] É. Cartan, Sur les variétés à connexion projective, Bull. Soc. Math. France 52 (1924) 205-241.

[4] M. Crampin, The second-order ordinary differential equation: Cartan, Douglas, Berwald, Period. Math. Hungarica 48 (2004) 151-164.

[5] M. Crampin, D.J. Saunders, On projective connections: the general case Preprint Department of Mathematical Physics and Astronomy, Ghent University, 2004. Available at http://maphyast.ugent.be.

[6] J. Douglas, The general geometry of paths, Ann. Math. 29 (1927/1928) 143-168.

[7] S. Fritelli, C. Kozameh, E.T. Newman, Differential geometry from differential equations, Comm. Math. Phys. 223 (2001) 383-408.

[8] E.T. Newman, P. Nurowski, Projective connections associated with second order ODEs, Class. Quant. Gravity 20 (2003) 2325-2335.

[9] P. Nurowski, G.A.J. Sparling, Three-dimensional Cauchy-Riemann structures and second-order ordinary differential equations, Class. Quant. Gravity 20 (2003) 4995-5016.

[10] R.W. Sharpe, Differential Geometry, Springer, 1997.

[11] Z. Shen, Differential Geometry of Spray and Finsler Spaces, Kluwer Academic Publishers, 2001 (Chapter 13). 